\title{
Biography of Prof. Dr. Hans-Martin Vieth
}

\author{
Gerd Buntkowsky $\cdot$ Konstantin L. Ivanov • \\ Hans-Heinrich Limbach • Ernst A. Rössler • \\ Alexandra V. Yurkovskaya
}

Received: 9 September 2012/Published online: 16 October 2012

(C) Springer-Verlag Wien 2012

Hans-Martin Vieth was born in a pastor's family in 1942 in Hannover, Germany, where he attended grammar school learning Latin and Greek, but little of natural sciences. Nevertheless, he decided to study physics at the nearby Technical University of Hannover in 1962. After his successful first two years he moved to Ruprecht-Karl-University, Heidelberg. He did his diploma work in 1967 at the MaxPlanck Institute of Nuclear Physics under the guidance of R. Jahr on "Spin-Flips during Inelastic Scattering of Deuterons on ${ }^{26} \mathrm{Mg},{ }^{58} \mathrm{Ni}$ and ${ }^{60} \mathrm{Ni}$ ". As he was fascinated already by this work by magnetic properties of elementary particles he continued his doctoral work at the Max-Planck Institute of Medical Research, Heidelberg, supervised by Karl-Hermann Hausser, a pioneer in magnetic resonance. He defended in 1973 his doctoral thesis entitled "Electron-Electron-Double Resonance of Nitroxide Radicals in Solution". In this work, he improved this electron-electron double resonance (ELDOR) technique using a novel microwave bridge and described its physics using Redfield's relaxation theory based on the density matrix formalism. The excellent agreement between experiment and theory allowed him to show that slow motions affect intramolecular and fast motions intermolecular relaxation. This work formed him as a scientist who knew and could apply the fundamentals of magnetic resonance to any system out of many that he

\footnotetext{
G. Buntkowsky

Eduard-Zintl-Institut für Anorganische und Physikalische Chemie,

Technische Universität Darmstadt, 64287 Darmstadt, Germany
}

K. L. Ivanov · A. V. Yurkovskaya

International Tomography Center, 630090 Novosibirsk, Russia

H.-H. Limbach

Institut für Chemie und Biochemie, Freie Universität Berlin, 14195 Berlin, Germany

E. A. Rössler $(\bowtie)$

Universität Bayreuth, Experimentalphysik II, 95440 Bayreuth, Germany

e-mail: ernst.roessler@uni-bayreuth.de 
studied later. In Heidelberg, HMV got acquainted and worked together with wellknown scientists in the field of magnetic resonance, as (alphabetically) Hermann Brunner, Klaus-Peter Dinse, Franz Fujara, Ulrich Haeberlen, Hans-Robert Kalbitzer, Paul Rösch, Dieter Schweitzer, Hans-Wolfgang Spiess, Dietmar Stehlik, Herbert Zimmermann and many others.

His curiosity for new countries and research field made him join the IBM Research Laboratory, San Jose, CA, USA in 1975, where he worked with Nino (Costantino S.) Yannoni in the field of solid-state nuclear magnetic resonance (NMR). He proposed a transient nutation experiment to remove dipolar splittings for efficient line narrowing in polycrystalline solids. Although HMV stayed only for one year, it was the beginning of a long-term collaboration with IBM. He returned often to IBM for sabbaticals to work with Nino on the efficiency of crosspolarization, with Nino and Dan Rugar on the force detection of magnetic resonance on surfaces, and with Anne S. Verhulst on quantum computing.

After coming back to Heidelberg, HMV combined his interests in both electron and nuclear magnetism and obtained already in 1977 a position as a Professor of Experimental Physics at the Freie Universität Berlin (FU-Berlin). In 1979, HMV described together with Volker Macho and Dietmar Stehlik, a new technique which uses optical nuclear polarization (ONP) permitting NMR detection of both the ground and the excited triplet states of molecules embedded in a crystalline matrix. He applied the technique to the "characterization of a photochemical hydrogen abstraction product found in fluorene single crystals doped with acridine or anthracene acting as hydrogen acceptors after optical excitation. In particular, hyperfine coupling constants of the transferred hydrogen could be determined. Satisfactory evidence can be given for the local structure of the molecular groups involved in the solid-state hydrogen transfer reaction" (J. Phys. Chem. 83: 3435-3440, 1979). This technique allowed HMV and his colleagues to study a number of reactive triplet states and their reactions in organic solids. Later, HMV introduced time-dependent ONP to follow the reactions in real time and to study mobility in single crystals. Coherent versus incoherent polarization transfer to nuclei was observed and analyzed, and used to enhance the sensitivity of ${ }^{13} \mathrm{C}$ and ${ }^{2} \mathrm{H}$ solid-state NMR. With his doctoral student Gerd Buntkowsky, now Darmstadt, he developed new multiple quantum NMR methods and techniques for ONP.

In order to develop his interests in solid-state NMR in all of its facets, he set up in the early 1980's a solid-state NMR laboratory in Berlin. As with IBM, many colleagues sought his collaboration and advice in order to solve a variety of problems. Again, he did not collaborate only with local colleagues in Berlin, but spent much of his free research time in Israel, USA and Russia; and once a collaboration was started, it continued for a long time, sometimes up to today.

With Shimon Vega and Zeev Luz (Rehovot) and later with Alexander M. Panich and Shaul D. Goren (Beer Sheva) he worked on zeolites, solid carbon from modified graphite to modified "detonation nanodiamond". From Igor V. Murin, St. Petersburg, came Alexei F. Privalov to Berlin to work with HMV on the dynamics of ion motions in superionic crystals as observed by NMR. The structure and dynamics of superconducting solids, from fluorinated yttrium-barium-copper-oxides up to 
doped fullerenes, were subject of a long-term collaboration with Klaus Lüders (FUBerlin).

HMV was and is as much interested in organic systems. He collaborated with Hans-Heinrich Limbach, Berlin, to elucidate very fast proton transfer rates in organic solids from longitudinal ${ }^{15} \mathrm{~N}$ and ${ }^{2} \mathrm{H}$ relaxation time measurements and to detect and analyze coherent and incoherent quantum tunneling of dihydrogen in organometallic solids. He helped organic chemists Jörg H. Fuhrhop and Gerhard Kossmehl (both FU-Berlin) to understand soft matter including molecular assemblies as well as liquid crystalline polymers, mainly studied using ${ }^{2} \mathrm{H}$ NMR: A particularly successful research was done by HMV with Ernst Rössler, now Bayreuth, on organic glasses. Their local structure was probed, in particular, using ${ }^{2} \mathrm{H}$ NMR of deuterated guest molecules such as benzene or hexamethyl benzene, whose re-orientation senses the variation of the local structure.

However, HMV never lost interest in the combination of electron and nuclear magnetism. Over the years, various techniques were devised and applied. It is then not astonishing, that he went back to the liquid solutions of his doctoral thesis, however, not to radicals but to radical pairs created by photoexcitation via triplet states, leading to chemically induced dynamic nuclear polarization (CIDNP). He has been working in this field till now together with Alexandra Yurkovskaya and Olga Morozova (Novosibirsk) and managed to obtain new information on short-lived radical pairs and biradicals by investigation of CIDNP kinetics and magnetic field dependence. HMV also had a successful collaboration with Hanns Fischer (Zurich) and Michael PaddonRow (Sydney) on electron exchange interaction in rigid biradicals. For doing these pioneering experiments HMV and his co-workers have developed a unique device, which enables fast field-cycling keeping high NMR resolution.

Later, HMV's run for perfection and true understanding of experimental data led to the discovery of new features concerning the dependence of CIDNP on strength of the magnetic field. At first sight, these features first looked as minor inconsistencies between the data and so far widely accepted theoretical concepts. With Alexandra Yurkovskaya and Konstantin Ivanov (Novosibirsk), HMV has convincingly shown that at low-field CIDNP is extremely efficiently redistributed in the reaction products due to scalar coupling of spins; moreover, it was shown that this process is coherent and new sharp features in the CIDNP field dependence have been found and explained as level anti-crossing effect. A similar concept was later applied to para-hydrogen-induced polarization (PHIP) where scalar couplings play a crucial role in re-distribution of hyperpolarization in the molecules studied. The full PHIP magnetic field dependence was for the first time measured and modeled theoretically in HMV's group. Surprisingly, remarkably strong effects of J-couplings also on the longitudinal relaxation of multi-spin systems were found, a circumstance which has been completely ignored for decades. HMV's group has also done pioneering works on pulsed overhauser-type dynamic nuclear polarization (DNP) showing that one can efficiently use coherent motion of electron spins to enhance the NMR signals of the nuclei. HMV's work in the field of spin hyperpolarization is recognized worldwide. He is probably the only person who is an expert in all known hyperpolarization techniques such as spin-exchange optical pumping, DNP, ONP, CIDNP and PHIP. He was a coordinator from the German 
side of the joint Russian-German workshop on spin hyperpolarization in 2010, where a European Cooperation In Science And Technology (COST) in the field of hyperpolarization was initiated.

HMV did a lot for promoting the Russian-German and Russian-EU cooperation. This was particularly valuable for the Russian side because the Russian science was in an extremely unfavorable position after the collapse of the Soviet Union. HMV's support allowed his Russian co-workers to run several exciting joint scientific projects. He coordinated two large projects involving groups from Germany, Russia, UK, Denmark and Austria, sponsored by the International Association for the Promotion of Cooperation with Scientists from the independent States of the former Soviet Union (INTAS). He was a host of four Humboldt fellows, one Marie Curie fellow and a few DAAD fellows coming from Russia. Within the framework of these projects HMV actively cooperated with Kev Salikhov (Kazan), Renad Sagdeev (Novosibirsk), Nikita Lukzen (Novosibirsk), Anatoly Buchachenko (Moscow), Peter Hore (Oxford), Guenter Grampp (Graz), Boiden Pedersen (Odense) and others.

Hans-Martin Vieth is a wonderful friend and colleague for all of us, has prevented us from falling into traps with his great knowledge of magnetic resonance; he has proposed us decisive experiments to solve our problems. He has been working in many different fields of magnetic resonance and in each of them he did truly unique and pioneering experiments. He is also known as a person who asks many questions, which often cannot easily be answered. In all cases these questions are aimed at going deep in all details to fully understand the physics underlying the observations. It happens very often that HMV's questions lead his co-workers to the right track and result in discovering new effects, which were ignored for a long time. All of his co-workers learned from him how to be honest in science, to be especially critical to own results and interpretations and to be open for new ideas.

Besides his achievements as scientist, HMV is an excellent teacher. He has been a Professor of Physics, who has trained several generations of students in this field as well as graduate students in magnetic resonance. As his sister Renate is a secondary school teacher in Hannover, HMV knew, in particular how important it is to train students who become later teachers of young pupils. Thus, HMV set up a laboratory and special experiments to train these teacher students, to bring over to them interest and enthusiasm for physics. He is a person from whom a young researcher can learn how to work in a physics laboratory, how to design and run experiments and how to avoid pitfalls. Not only in science, his broad knowledge in history, politics and geography is continuing to impress us. His readiness to discuss political issues can be witnessed, for example, each year on the NMR meeting gathering several NMR groups where in the evening talks non-scientific topics are on the agenda. Moreover, HMV was involved in a program where he regularly visited grammar schools to show young pupils the way how new insight into physical problems is achieved. HMV received many graduate students and postdocs from different countries, in particular from Russia and helped them to start a career in magnetic resonance.

We wish Hans-Martin Vieth an excellent health that he will be able to continue his fruitful work as long as possible. We are looking forward for his new results and cooperation with him. 